18. Let $I_{n}{ }^{\prime}, I_{n}{ }^{\prime \prime}, I_{n}{ }^{\prime \prime \prime}$ be the three points of inflection of $\Gamma_{n}$. The lines $O I_{n}{ }^{\prime}, O I_{n}{ }^{\prime \prime}, O I_{n}{ }^{\prime \prime \prime}$ meet every cubic of the pencil $\Gamma$ in three collinear points (14a), and all these points are points of inflection (17). Consequently: The points of inflection of all the cubics of the pencil $\Gamma$ lie on three straight lines concurring in the double point.

19. From (18) and (14b) it follows: The inflectional lines of the cubics of the pencil $\Gamma$ form a pencil having its vertex on the basic line.

It may be added that this vertex is the harmonic conjugate, with respect to the couple $A, A_{1}$, of the trace on $A B C$ of the locus of the tangentials of $A(4)$.

UNIVERSITY OF OKLAHOMA, October, 1919.

\title{
DEFINITION AND ILLUSTRATIONS OF NEW ARITHMETICAL GROUP INVARIANTS.
}

BY PROFESSOR E. T. BELL.

1. Arithmetical instances of groups are still sufficiently uncommon to make any new occurrence a matter of interest. Many significant group concepts have, of course, been implicit in arithmetic since at least the times of Euler and Gauss, notably in the theories of power residues, the automorphics of binary quadratic forms, and principal genera. More recently Miller has directly applied groups to quadratic residues and other topics.

This note contains the definition and a few illustrations, shorn of algebraic and other details, of certain group invariant relations for arbitrary integers, which are believed to be fundamentally distinct from previous group phenomena in arithmetic. These relations are genuinely arithmetical in that they concern only integers, and they may legitimately be called group relations because they exist only in reference to groups.

The object of this note is merely to call attention to these invariants by exhibiting a few of the simplest; and as several preliminary definitions are required - the subject being newdevelopments and less obvious examples may be left to another 
occasion. Although the illustrations have purposely been chosen of a very simple nature, nevertheless they sufficiently illustrate the general character of all such results, and notes on their analytical origins have been included. We point out here, however, that the elliptic, abelian, and allied theta functions offer inexhaustible mines of such invariants for systems of any number of quadratic and bilinear forms and for groups of any degree, and that applications of these invariants to numerous arithmetical problems are immediately apparent. For example, it will be clear from this note that any relation between theta functions and their allied quotients, provided only that it involves the arguments and is not merelv an identity between constants, gives rise to at least one singular monad invariant, and therefore to several dyads, triads, etc. Regarding applications, the possible fruitfulness of the invariants will be evident on remarking that in any invariant the $k$-ads may be replaced by arbitrary functions of the same parity as the $k$-ads and belonging to the same group; so that each $k$-ad relation implies an infinity of specialized arithmetical theorems. Last, the whole method is obviously applicable to functions other than the thetas as a basis, provided they are expansible in Fourier series. But so far the most interesting applications have arisen by way of the theta functions.

2. The sets of independent variables, in which order is essential, $\left(x_{1}, x_{2}, \cdots, x_{a}\right),\left(-x_{1},-x_{2}, \cdots,-x_{a}\right)$, are denoted by by $\xi,-\xi$ respectively. Consider $(r+s)$ such sets, all the variables being independent,

$$
\begin{array}{ll}
\xi_{i} \equiv\left(x_{i 1}, x_{i 2}, \cdots, x_{i a_{i}}\right), & (i=1,2, \cdots, r), \\
\eta_{j} \equiv\left(y_{j 1}, y_{j 2}, \cdots, y_{j b_{j}}\right), & (j=1,2, \cdots, s),
\end{array}
$$

united in a symbol $Z$, in which order is essential,

$$
Z \equiv\left(\xi_{1}, \xi_{2}, \cdots, \xi_{r} \mid \eta_{1}, \eta_{2}, \cdots, \eta_{s}\right),
$$

which is called an $(r+s)$-ad, of parity and order

$$
\left(a_{1}, a_{2}, \cdots, a_{r} \mid b_{1}, b_{2}, \cdots, b_{s}\right), \quad \omega \equiv \sum_{i=1}^{r} a_{i}+\sum_{j=1}^{s} b_{j}
$$

respectively, and which has the following properties: $Z$ exists and is uniquely determined when all the variables take integral values 0 , remains equal to itself when any $\xi$ is replaced 
by its negative, changes sign for a like replacement of any $\eta$, and vanishes when all the variables in any $\eta$ take zero values simultaneously. Likewise, by a few changes in the wording, we define the $(r+0)$-ad $Z^{\prime}$ and the $(0+s)$-ad $Z^{\prime \prime}$,

$$
Z^{\prime} \equiv\left(\xi_{1}, \xi_{2}, \cdots, \xi_{r} \mid\right), \quad Z^{\prime \prime} \equiv\left(\mid \eta_{1}, \eta_{2}, \cdots, \eta_{s}\right),
$$

of the obviously corresponding parities and orders $\left(a_{1}, a_{2}\right.$, $\left.\cdots, a_{r} \mid\right), \Sigma a_{i}$, etc. If $r+s=k, r+s>0$, we shall speak of $Z, Z^{\prime}, Z^{\prime \prime}$ simply as $k$-ads.

All the formal laws of algebraic addition and subtraction are to hold for $k$-ads; and two $k$-ads are equal when and only when it is possible, by legitimate changes of sign in accordance with the foregoing definitions, to reduce them to identically the same form, the signs prefixed to the whole $k$-ads being included. Thus $(2,3 \mid),(-2,3 \mid),(2,-3 \mid),(-2,-3 \mid)$, are equal; $(2,3 \mid)$ and $(3,2 \mid)$ are unequal, as also are $((2,3) \mid)$ and $(2,3 \mid) ;(\mid(2,3))=-(\mid(-2,-3)) ;$ and

$$
\begin{aligned}
& (1,(-2,0,3) \mid(-4,0), 5)=(1,(2,0,-3) \mid(-4,0), 5) \\
= & -(-1,(-2,0,3) \mid(4,0), 5)=(1,(-2,0,3) \mid(4,0),-5) .
\end{aligned}
$$

By the variables of a $k$-ad we shall mean the variables within the sets $\xi, \eta$, and not the $\xi$, $\eta$ which will be called the sets of the $k$-ad.* If each set contains but one letter, the sets and the variables coincide.

3. Let $1, s_{1}, s_{2}, \cdots, s_{t} \equiv g$ denote a set of substitutions on some or all of the variables of a $k$-ad $Z$, and write $\left(s_{i} Z\right)$ for the result of applying $s_{i}$ to $Z$. The $s_{i}$ fall into two categories: $(A)$, substitutions which either permute the variables of $Z$ within the sets, interchanging no pair of variables from different sets, or permute the sets $\xi$ among themselves and the sets $\eta$ among themselves, interchanging no $\xi$ with any $\eta$; $(B)$, all others. If all the substitutions of a group $G$ belong to $(A)$, we call $G$ an $A$-group, ("for $Z$ " being understood). In this note we consider only $A$-substitutions and $A$-groups, as the consequences are simpler than those arising from $(B)$.

If $g$ is any $A$-group on the variables of $Z$, we shall denote by $Z^{g}$ any $k$-ad of the same parity as $Z$, and on the same variables, which belongs to $g$. As a simple example, the following sum is a $(1+0)$-ad of parity $(4 \mid)$ and order 4 :

* This is emphasized because in one application of the theory the $\xi, \eta$ behave as single variables, not as sets, and are then treated as the independent variables. 


$$
\begin{array}{r}
(x \mid y,(z, w))+(x \mid w,(z, y))+(y \mid z,(w, x))+(y \mid x,(w, z)) \\
+(z \mid w,(x, y))+(z \mid y,(x, w))+(w \mid x,(y, z))+(w \mid z,(y, x))
\end{array}
$$

which obviously is invariant for an octic group $g$ on $x, y, z, w$ and which therefore is a particular monad of that whole class any member of which is denoted by $((x, y, z, w) \mid)^{g}$. As another example, if $\lambda, \mu$ denote integers different from zero, and $\lambda$ is even, $\mu$ odd, the function

$$
x^{\lambda}\left(y^{\mu}-z^{\mu}\right)+y^{\lambda}\left(z^{\mu}-x^{\mu}\right)+z^{\lambda}\left(x^{\mu}-y^{\mu}\right)
$$

belonging to $g \equiv 1, x y z, x z y$, is of parity $(\mid 3)$ in $(x, y, z)$ and is therefore a particular $(\mid(x, y, z))^{g}$ for this $g$. Last, recalling a common notation, we denote by $[\alpha \beta \gamma \delta],[\alpha \beta \gamma \delta]^{\prime}$ the products of any four elliptic theta functions $\vartheta_{a}(x)$, etc., in which the variables are, for the order in which the functions are written, $x, y, z, w$ and $x^{\prime}, y^{\prime}, z^{\prime}, w^{\prime}$ respectively, where

$$
\begin{gathered}
2 s \equiv x+y+z+w, \\
x^{\prime}=s-x, \quad y^{\prime}=s-y, \quad z^{\prime}=s-z, \quad w^{\prime}=s-w ;
\end{gathered}
$$

and we have either of the equal sums

$$
\begin{gathered}
{[0123]+[1032]+[2301]+[3210],} \\
{[0123]^{\prime}+[1032]^{\prime}+[2301]^{\prime}+[3210]^{\prime}}
\end{gathered}
$$

a particular $(\mid(x, y, z, w))^{g}$ for $g$ the four group $1, x y \cdot z w$, $x z \cdot y w, x w \cdot y z$. We interpret this example arithmetically later. Although all of these are for monads, it is not difficult to devise similar examples for dyads, triads, etc. We pass over these, since from invariant relations concerning monads may be inferred others involving dyads, etc., as pointed out in $\S 12$.

4. Consider a $k$-ad $Z$ whose variables are $z_{1}, z_{2}, \cdots, z_{\omega}$, and an $A$-group $g \equiv 1, s_{1}, s_{2}, \cdots, s_{r}$ on some (or all) of these variables, and let

$$
S:\left(z_{1}, z_{2}, \cdots, z_{\omega}\right) \equiv\left(z_{1}{ }^{(i)}, z_{2}{ }^{(i)}, \cdots, z_{\omega}{ }^{(i)}\right),(i=1, \cdots, t)
$$

denote a system of $t$ integral values of the set $\left(z_{1}, z_{2}, \cdots, z_{\omega}\right)$; viz., each $z_{j}{ }^{(i)}$ is an integer 0 , and in the typical identity between sets just written, $z_{j}=z_{j}{ }^{(i)}(j=1,2, \cdots, \omega)$. Replace $z_{j}$ by $z_{j}{ }^{(i)}$ in $Z,(j=1,2, \cdots, \omega)$, multiply* the result

* From $\$ 2$ the meaning of $c Z$, where $c$ is an integer, is $Z+Z+\cdots$ $+Z$ ( $c$ terms) if $c>0$; it is $-Z-Z-\cdots-Z$ if $c<0$; and $c Z=0$ when $c=0$. 
by an integer $c_{i}$, form the sum of all such products for $i=1,2$, $\cdots, t$, and denote the result by $\int_{8} c Z$, or by $\int c Z$ where $S$ is understood. In the same way are defined $\int c\left(s_{i} Z\right), \int c Z^{g}$, starting from $\left(s_{i} Z\right)$ and $Z^{g}$ respectively. In these sums the substitutions $s_{i}$ and those of $g$ must obviously be applied before the variables of $Z$ are replaced by the integers of $S$.

5 . In all that follows there is a radical distinction between special and general sums of the sort just defined. In a special sum the integers $z_{j}{ }^{(i)}$ are constants, $0,-2,5, \cdots$, etc.; in a general sum they are variables. Through several of the $z_{j}{ }^{(i)}$ having equal absolute values we frequently find $\int c Z=0$ for $Z$ special, but $\int c Z \neq 0$ for $Z$ general; and so in the other cases. Henceforth all $\int$ sums are general.

6 . Bearing in mind that the $\int$ are general, we readily see that relations

$$
\int c Z=0, \quad \int c\left(s_{i} Z\right)=0, \quad \int c Z^{o}=0,
$$

must be mere trivial identities until the integers of $S$ are in some way restricted. Hence we next impose conditions upon $S$, emphasizing again, however, that within the restrictions the integers of $S$ are variables. For example let $\left(x_{i}, y_{i}, z_{i}, w_{i}\right)$, $(i=1, \cdots, h)$ represent the totality of sets of four integers $>0$, the sum of whose squares is equal to an odd integer $m$, and choose

$$
S:\left(z_{1}, z_{2}, z_{3}, z_{4}\right) \equiv\left(x_{i}, y_{i}, z_{i}, w_{i}\right) \quad(i=1, \cdots, h) .
$$

Then in this case our sums are to refer to $S$ for $m$ an arbitrary odd positive integer; and we shall seek for this $S$ a relation such as $\int c\left(\left(z_{1}, z_{2}, z_{3}, z_{4}\right) \mid\right)^{g}=0$, valid for any odd positive integer whatever.

7. It follows at once from the definitions that if $g$ is an $A$-group, and $s_{i}$ a substitution of $(A)$, then the first of the $\int$ relations in $\S 6$, wherein $S$ is now restricted, implies each of the others. For $Z^{g}$ is a special case of $Z$; and $\int c\left(s_{i} Z\right)=0$ is $\int c Z=0$ with a changed notation. E.g., if $Z \equiv\left(\left(z_{1}, z_{2}, z_{3}, z_{4}\right) \mid\right)$ as above, and $s_{1}=z_{1} z_{3} \cdot z_{2} z_{4}, \int c\left(\left(z_{1}, z_{2}, z_{3}, z_{4}\right) \mid\right)=0$ becomes, on applying $s_{1}, \int c\left(\left(z_{3}, z_{4}, z_{1}, z_{2}\right) \mid\right)=0$, which abstractly is the same thing; and similarly for $A$-substitutions, which interchange sets. Clearly the like does not hold for $B$-substitutions.

Suppose now that $\int c Z=0$. Then this implies $\int c Z^{\theta}=0$, as remarked, and this is an invariant relation for the $A$-group 
g. We shall call this an improper invariant, it being included as a very special case in another relation, $\int c Z$, between $k$-ads on the same variables. We proceed to define proper invariants of $S$ for $g$, stating the definition for the general case. These express essential invariant properties of $S$; the improper, considered as invariants, are trivial, the relations from which they follow being more general.

8. Let $F_{i}(i=1, \cdots, k)$ denote a system of $k$ algebraic forms, not necessarily all of the same orders and degrees, with integral coefficients, and consider all the representations of a fixed arbitrary integer $n$ in the system $\Sigma$,

$$
\Sigma: \quad F_{i}=n \quad(i=1, \cdots, k) .
$$

Consider first only one of the equations in $\Sigma$; say it is $F\left(w_{1}, w_{2}, \cdots, w_{h}\right)=n$, and suppose it to have precisely $t$ distinct solutions. ${ }^{*}$ Let

$$
\left(w_{i}\right) \equiv\left(w_{1}^{(i)}, w_{2}{ }^{(i)}, \cdots, w_{h}^{(i)}\right), \quad(i=1,2, \cdots, t),
$$

be these solutions. From the integers in one of them, $\left(w_{i}\right)$, construct $\omega$ linear homogeneous functions $\lambda$ with constant rational coefficients, some of which may be zeros,

$\lambda_{p}{ }^{(i)} \equiv a_{p 1} w_{1}{ }^{(i)}+a_{p 2} w_{2}{ }^{(i)}+\cdots+a_{p h} w_{h}{ }^{(i)},(p=1,2, \cdots, \omega)$.

With the notation at the beginning of $\S 4$ choose

$\left(z_{1}{ }^{(i)}, z_{2}{ }^{(i)}, \cdots, z_{\omega}{ }^{(i)}\right) \equiv\left(\lambda_{1}{ }^{(i)}, \lambda_{2}{ }^{(i)}, \cdots, \lambda_{\omega}{ }^{(i)}\right),(i=1,2, \cdots, t)$;

make this substitution in $\int c Z, \int c\left(s_{i} Z\right), \int c Z^{g}$ as there $(\S 4)$ defined, and denote the results by

$$
\int c \lambda, \quad \int c\left(s_{i} \lambda\right), \quad \int c \lambda^{g} .
$$

Returning to the system $\Sigma$, we proceed similarly with all of its equations, forming $\lambda$ 's for each, the coefficients in the $\lambda$ 's for the several forms $F_{j}$ not necessarily being the same, and getting finally the sums

$$
\int_{j} c^{(j)} \lambda, \quad \int_{j} c^{(j)}\left(s_{i} \lambda\right), \quad \int_{j} c^{(j)} \lambda^{g}, \quad(j=1, \cdots, k),
$$

where the suffix $j$ signifies that the sums belong to the particular equation $F_{j}=n$, and $j$ in $c^{(j)}$ distinguishes the constants in the several $\int$ sums. Next sum each of these for

* It is immaterial whether $t$ is finite or infinite. In this note we illustrate only invariants for $\Sigma$ 's having a finite number of solutions. 
$j=1$ to $k$, that is, over the entire system $\Sigma$, and write the results

$$
\begin{aligned}
& \sum_{j=1}^{k} \int_{j} c^{(j)} \lambda, \quad \sum_{j=1}^{k} \int_{j} c^{(j)}\left(s_{i} \lambda\right), \quad \sum_{j=1}^{k} \int_{j} c^{(j)} \lambda^{g}, \\
& \equiv \int_{\Sigma} c \Lambda, \quad \int_{\Sigma} c\left(s_{i} \Lambda\right), \quad \int_{\Sigma} c \Lambda^{g},
\end{aligned}
$$

respectively, or the same with the omission of $\Sigma$ where it is understood.

Last, let $g \equiv 1, s_{1}, \cdots, s_{r}$ be a group whose substitutions are unrestricted with respect to $(A),(B)$ on some or all of the variables of the $Z$ from which $\Lambda$ is derived as above. Then, if it be possible to choose the coefficients of the $\lambda$ 's so that $\int c(s \Lambda) \neq 0$ for at least one* substitution $s$, which may be the identity, of $g$, while $\int c \Lambda^{g}=0$, the latter relation is defined to be a proper $g$-invariant relation, or simply $g$-invariant, of $\Sigma$.

9. It can be shown without difficulty that the first of the following implies the second,

$$
\int c \Lambda+\sum_{i=1}^{r} \int c\left(s_{i} \Lambda\right)=0, \quad \int c \Lambda^{g}=0,
$$

all the notation being as in $\S 8$. Indeed this is an immediate consequence of the definitions, and we may omit a formal proof. Again if $\gamma$ is any subgroup of $g$, all of whose substitutions not in $\gamma$ belong to $(A)$, it is easily seen that of the following the second is implied by the first,

$$
\int c \Lambda^{\gamma}=0, \quad \int c \Lambda^{g}=0 .
$$

The like does not hold if any substitution of $g$ not in $\gamma$ belongs to $(B)$. We shall say that the $g$-invariant is contained in the $\gamma$-invariant; and writing these for the moment $I_{g}, I_{\gamma}$ respectively, symbolize the inclusion thus, $I_{g}>I_{\gamma}$. If now $I_{\gamma}>I_{\delta}$, $I_{\delta}>I_{\beta}, \cdots, I_{\alpha}>I_{\mathrm{e}}$, we have

$$
I_{g}>I_{\gamma}>I_{\delta}>I_{\beta}>\cdots>I_{a}>I_{e},
$$

and clearly any such series of inclusions contains only a finite number of terms. If $I_{\mathrm{e}}$ is the last term, viz., if there is no group $\zeta$ different from $\epsilon$ such that $I_{\epsilon}>I_{\zeta}$, we call $I_{e}$ the reduced invariant of $I_{a}, I_{\beta}, \ldots, I_{g}$. Henceforth invariant means reduced invariant. When $\epsilon$ is the identity, $I_{\epsilon} \equiv I_{1}$ is called a unit.

* If $g$ is an $A$-group this implies $\int c(s \Lambda) \neq 0$ for every $s$ of $g$. 
10. With the notation of $\S 8$, let

$$
\sum_{j=1}^{k} \int_{j} c^{(j)} \lambda \equiv \int_{\Sigma} c \Lambda \equiv \int c \Lambda=0
$$

be a unit; and let $1, \sigma_{1}, \sigma_{2}, \cdots, \sigma_{k-1}$ denote a set of substitutions belonging to $(A)$ on the variables of the $Z$ from which this $I_{1}$ is derived. Write $\int c \Lambda=0$ in full,

and put

$$
\int_{1} c^{(1)} \lambda+\int_{2} c^{(2)} \lambda+\cdots+\int_{k} c^{(k)} \lambda=0,
$$

$$
I \equiv \int_{1} c^{(1)} \lambda+\int_{2} c^{(2)}\left(\sigma_{1} \lambda\right)+\cdots+\int_{k} c^{(k)}\left(\sigma_{k-1} \lambda\right) .
$$

We cannot infer $I=0$, that is, $I=0$ is not a unit invariant; but if the $\sigma$ are substitutions of an $A$-group $g$ which are not all contained in a group of order lower than that of $g$, then clearly $I=0$ is a $g$-invariant (proper and reduced) of $\Sigma$. We shall call $I=0$ a singular $g$-invariant of $\Sigma$. Several of the $F_{j}$ in $\Sigma$ may be identical. For such $\Sigma$ the unit invariant $\int c \Lambda=0$ appears in the form

$$
\int_{1} c^{(1)} \lambda+\int_{2} c^{(2)}\left(s_{1} \lambda\right)+\cdots+\int_{k} c^{(k)}\left(s_{k-1} \lambda\right),
$$

where the $s_{j}$ are substitutions on the variables of $Z$. As before, we get a singular $g$-invariant $J=0$, where

$$
J \equiv \int_{1} c^{(1)} \lambda+\int_{2} c^{(2)}\left(s_{1} \sigma_{1} \lambda\right)+\cdots+\int_{k} c^{(k)}\left(s_{k-1} \sigma_{k-1} \lambda\right),
$$

on choosing $s_{1} \sigma_{1}, s_{2} \sigma_{2}, \cdots, s_{k-1} \sigma_{k-1}$ so that they do not all occur in a group whose order is lower than that of $g$.

11. Taking any $g$-invariant for $\Sigma$ as a primitive, we deduce from it another $g$-invariant called the derivative of the primitive, as follows. Let $Z$ be the $k$-ad from which, as in $\S 8$, $\int c \Lambda^{g}=0$ is constructed, and let $\zeta_{1}, \zeta_{2}, \cdots, \zeta_{k}$ be the sets of $Z$. Call $f\left(\zeta_{i}\right)$ an even or an odd function of $\zeta_{i}$ according as $f\left(\zeta_{i}\right)=f\left(-\zeta_{i}\right)$ or $f\left(\zeta_{i}\right)=-f\left(-\zeta_{i}\right)$. In each set to the left of the bar in $Z$ replace each variable by an arbitrary odd or even function of the set in which it occurs, and in each set to the right of the bar in $Z$ replace each variable by an arbitrary odd function of the set in which it occurs, getting thus a new $k$-ad $Z^{\prime}$ which is of the same parity in the variables of $Z$ as is $Z$. Starting from $Z^{\prime}$ construct the sum $\int c \Lambda^{\prime \prime}$ in precisely the same way that $\int c \Lambda^{g}$ is constructed, viz., by substituting for the $z$-variables in $Z$ the $\lambda$ 's determined by $\Sigma$, and summing. 
Then clearly the first of the following implies the second, which is called the derivative of the first,

$$
\int_{c} \Lambda^{g}=0, \quad \int_{c} \Lambda^{\prime g}=0 .
$$

Clearly a primitive is neither more nor less general than its derivative. Nevertheless a derivative frequently suggests applications to specific problems more readily than does the primitive.

12. We write $\int((x, y) \mid)^{g}=0, \int((x, y, z) \mid)^{g}=0$, etc., understanding that the $\int$ sums refer to all $(x, y),(x, y, z), \cdots$, the $x, y, z, \cdots$ in any case being $\lambda$ 's determined as in $\S 8$ for a system $\Sigma$.

The number of implications increasing rapidly with the order of the $k$-ads, we shall write out the complete sets for orders 2,3 only.*

Consider now the following tables.

$$
\begin{array}{lll}
((x, y) \mid): & (x, y \mid), \quad(\mid x, y) \\
(\mid(x, y)): & (x \mid y), \quad(y \mid x) \\
((x, y, z) \mid): & (x,(y, z) \mid), \quad(\mid x,(y, z)) \\
& (y,(z, x) \mid), \quad(\mid y,(z, x)) \\
& (z,(x, y) \mid), \quad(\mid z,(x, y)) \\
(x, y, z \mid), \quad(x \mid y, z), \quad(y \mid z, x), \quad(z \mid x, y) \\
(\mid(x, y, z)): & (x \mid(y, z)), \quad((y, z) \mid x) \\
& (y \mid(z, x)), \quad((z, x) \mid y) \\
& (z \mid(x, y)), \quad((x, y) \mid z) ; \\
(\mid x, y, z), \quad(y, z \mid x), \quad(z, x \mid y), \quad(x, y \mid z) .
\end{array}
$$

The meaning of these will be seen from the first row of (III). It is: $\int((x, y, z) \mid)^{g}=0$ implies $\int(x,(y, z) \mid)^{g}=0$ and $\int(\mid x,(y, z))^{g}=0$; and inversely the last two together, but neither singly, imply the first. Similarly for the pairs and

* A systematic rule for deducing all the implications for order $k$ will be evident on reading $\S 9$, p. 319, of a paper in this BuLletin, vol. 25, 1919. A proof of the general theorem for units is given in the Introduction to Part 1 of "Arithmetical paraphrases," presented to the Society, Oct., 1918 , and the result for $I_{g}, g \neq 1$, follows at once from this. $\$ 13$ is also proved (in much more general form) in the same place. 
set of four in the remaining rows of (III); likewise for (I)-(IV). For a monad invariant of parity $(4 \mid), \delta((x, y, z, w) \mid)^{g}=0$ implies $\int(x,(y, z, w) \mid)^{g}=0$ and $\int(\mid x,(y, z, w))^{g}=0$; these together imply the original; and from (I)-(IV) the complete set of implications may be written out for order 4, parity (4|). For $\int(\mid(x, y, z, w))^{g}=0$ the first pair of implications is $\int(x \mid(y, z, w))^{g}=0, \int((y, z, w) \mid x)^{g}=0$; and the procedure for monads of parities $(k \mid),(\mid k)$ is evident. By the notation

$$
\iint((x, y, z) \mid)^{g}=0
$$

we shall mean the complete set of eleven invariants written down from (III) by replacing $((x, y, z) \mid)$ in $\int((x, y, z) \mid)^{g}=0$ by each of the eleven dyads and triads in (III), and similarly for order $k$.

13. It remains to indicate the connection of these invariants with the theta and allied functions. From any identity between such functions in the variables $\alpha, \beta, \cdots, \gamma$, we deduce trigonometric identities in $\alpha, \beta, \cdots, \gamma$, of which the following is one type,

$$
\Sigma c \cos (x \alpha+y \beta+\cdots+z \gamma)=0,
$$

the summation referring to the constants $c$ and the integers $x, y, \cdots, z$, the latter being the $\lambda$ 's of $\S 8$. It can be proved without difficulty, but at some length (cf. $\$ 12$, footnote) that this trigonometric identity implies $\int c((x, y, \cdots, z) \mid)=0$. If the identity involves sines instead of cosines, we get $\int(\mid(x, y, \cdots, z))=0$. Groups enter either ab initio in the irreducible form of the theta identity, giving rise to trigonometric identities that are invariants under substitutions on the $\alpha, \beta, \cdots, \gamma$, or in the derivation of singular from unit invariants as already indicated.

The following examples, the last of which is numerical, illustrate the principal definitions.

14. (i) As a first example let $\Sigma(\S 8)$ be the single equation

$$
2 m=l_{1}{ }^{2}+l_{2}{ }^{2}+m_{3}{ }^{2}+m_{4}{ }^{2},
$$

wherein $m$ is odd, $l_{1}, l_{2}$ even $0, m_{3}, m_{4}$ odd $\gtreqless 0$; write $(\mid(x, y, z, w))^{g} \equiv\{x, y, z, w\}$ where $g$ is the four group in $\S 3$; and

$$
2 \sigma \equiv l_{1}+l_{2}+m_{3}+m_{4}, \quad \lambda_{i} \equiv \sigma-l_{i}, \quad \mu_{i} \equiv \sigma-\mu_{i} .
$$


Then the theta identity in $\S 3$ leads to a trigonometric identity (on equating coefficients of $q^{2 m}$ ) which, as indicated in $\S 13$, paraphrases by means of $\S \S 13,12$ into the set of invariants

$$
\iint\left[\left\{l_{1}, m_{3}, m_{4}, l_{2}\right\}-\left\{\lambda_{1}, \mu_{3}, \mu_{4}, \lambda_{2}\right\}\right]=0 .
$$

(ii) Similarly, from one of Kronecker's forms of the "equation of three terms," * for $g \equiv 1, y z w, y w z$, and $((x, y, z, w) \mid)^{g}$ $\equiv\{x, y, z, w\}$ we get when $\Sigma$ is the equation

$$
4 n=m_{1}{ }^{2}+m_{2}^{2}+m_{3}{ }^{2}+m_{4}{ }^{2},
$$

where $m_{1}, m_{2}, m_{3}, m_{4}$ are odd $\gtrless 0$, on writing $2 \mu \equiv m_{1}+m_{2}$ $+m_{3}+m_{4}$, the invariants

$$
\iint(-1)^{\mu}\left\{m_{1}+m_{2}, m_{1}-m_{2}, m_{3}+m_{4}, m_{3}-m_{4}\right\}=0 .
$$

(iii) From one of Briot and Bouquet's forms of the same equation, $\dagger$ we find for the same $\Sigma, g$, the invariants $\iint(-1)^{\alpha}\left\{m_{1}+m_{3}, m_{2}+m_{3}, m_{1}+m_{2}, m_{3}+m_{4}, m_{3}-m_{4}\right\}=0$, where now $((u, v, y, z, w) \mid)^{g} \equiv\{u, v, y, z, w\} ; \alpha$ is either $\mu$ or $\sigma$ and these are

$$
2 \mu \equiv m_{1}+m_{2}+m_{3}+m_{4}, \quad 2 \sigma \equiv m_{3}+m_{4} .
$$

(iv) From the same source, for $\Sigma$ as in (i), and $g,\{u, v, y$, $z, w\}$ as in (iii),

$2 \rho \equiv l_{1}+l_{2}+m_{3}+m_{4}, 2 \sigma \equiv m_{3}+m_{4}, 2 \nu+1 \equiv l_{1}+m_{4} ;$

$\iint(-1)^{\beta}\left\{l_{1}+m_{3}, l_{2}+m_{3}, l_{1}+l_{2}, m_{3}+m_{4}, m_{3}-m_{4}\right\}=0$,

where $\beta$ is $\rho, \sigma$ or $\nu$.

(v) From the same source, for $\Sigma$ the system of three equations, $m$ odd,

$$
2 m=u_{1}{ }^{2}+v_{1}{ }^{2}=x_{2}{ }^{2}+u_{2}{ }^{2}+v_{2}{ }^{2}=x_{3}{ }^{2}+y_{3}{ }^{2}+u_{3}{ }^{2}+v_{3}{ }^{2},
$$
$u_{1}, v_{1}, u_{2}, v_{2}, u_{3}, v_{3}$ odd $\gtrless 0, x_{2}, x_{3}, y_{3}$ even $\gtrless 0$, and $g$, \{\} as in (iii), $2 \mu_{i}=u_{i}+v_{i}$, we find

$$
\begin{aligned}
& \iint\left[(-1)^{\mu_{1}}\left\{u_{1}, 0, u_{1}, v_{1},-v_{1}\right\}\right. \\
& \left.\quad+(-1)^{\mu_{3}}\left\{y_{3}+u_{3}, x_{3}+y_{3}, x_{3}+u_{3}, y_{3}+v_{3}, y_{3}-v_{3}\right\}\right] \\
& +\iint(-1)^{\mu_{2}}\left[\left\{u_{2}, x_{2}, x_{2}+u_{2}, v_{2},-v_{2}\right\}\right. \\
& \left.\quad+\left\{x_{2}+u_{2}, x_{2}, u_{2}, x_{2}+v_{2}, x_{2}\right\}\right]=0 .
\end{aligned}
$$

*Crelle, vol. 102, p. $262(F)$.

$\dagger$ Théorie des Fonct. ellip. (éd. 2, 1875), p. 488. 
(vi) The identity, with $\varphi_{1}(x, y) \equiv \vartheta_{1}{ }^{\prime} \vartheta_{1}(x+y) / \vartheta_{0}(x) \vartheta_{0}(y)$,

$$
\vartheta_{0}(x) \vartheta_{0}(y) \vartheta_{0}(z) \varphi_{1}(x, y)=\vartheta_{1}^{\prime} \vartheta_{0}(z) \vartheta_{1}(x+y) \text {, }
$$

on expanding $\varphi_{1}(x, y)$ in a Fourier series and paraphrasing as outlined in (i), gives invariants for another kind of $\Sigma$, viz.,

$$
2 m=x_{1}^{2}+y_{1}^{2}+z_{1}^{2}+.2 u_{1} v_{1}=x_{2}^{2}+u_{2}^{2}+v_{2}^{2},
$$

where $x_{1}, y_{1}, z_{1}, x_{2}$ are even $0, u_{1}, v_{1}, u_{2}, v_{2}$ odd, $u_{1}, v_{1}>0$, $u_{2}, v_{2} \gtrless 0$;

$$
\iint\left[4(-1)^{a}\left\{x_{1}+u_{1}, z_{1}, y_{1}+v_{1}\right\}+(-1)^{\beta}\left\{u_{2}, x_{2}, u_{2}\right\}\right]=0,
$$

where $\{x, y, z\} \equiv(\mid(x, y, z))^{g}, g \equiv 1, x y z, x z y$, and

$$
2 \alpha \equiv x_{1}+y_{1}+z_{1}, \quad 2 \beta \equiv x_{2}+u_{2}+v_{2} .
$$

(vii) From (vi) we get, in the same notation except $g$, the following invariants for $g$ the symmetric group on $x, y, z$,

$$
\iint\left[4(-1)^{a}\left\{x_{1}+u_{1}, y_{1}+v_{1}, z_{1}\right\}+(-1)^{\beta}\left\{u_{2}, u_{2}, x_{2}\right\}\right]=0 \text {. }
$$

(viii) Putting, for the $m$ 's odd $\gtreqless 0$, the $l$ 's even $\gtreqless 0$,

$$
\begin{gathered}
\mu_{1}=m_{1}+m_{2}, \quad \mu_{2}=m_{3}+m_{4}, \quad \mu_{3}=m_{3}-m_{4}, \\
\lambda_{1}=l_{1}+l_{2}, \quad \lambda_{2}=l_{3}+l_{4}, \quad \lambda_{3}=l_{3}-l_{4}, \\
\mu_{4}=m_{1}+m_{3}, \quad \mu_{5}=m_{2}+m_{3}, \\
\lambda_{4}=l_{1}+l_{3}, \quad \lambda_{5}=l_{2}+l_{3},
\end{gathered}
$$

we get (from the same source as (iii)) singular invariants for the system

$$
\begin{gathered}
4 n=m_{1}{ }^{2}+m_{2}{ }^{2}+m_{3}{ }^{2}+m_{4}{ }^{2}=l_{1}{ }^{2}+l_{2}{ }^{2}+l_{3}{ }^{2}+l_{4}{ }^{2}, \\
2 \mu \equiv m_{1}+m_{2}+m_{3}+m_{4},
\end{gathered}
$$

on writing $\{x, y, z, u, v\} \equiv((x, y, z, u, v) \mid)^{g}$, as follows:

$$
\begin{aligned}
& \iint\left[(-1)^{\mu}\left\{\mu_{3}, \mu_{2}, \mu_{1}, \mu_{4}, \mu_{5}\right\}+\left\{\lambda_{3}, \lambda_{4}, \lambda_{2}, \lambda_{1}, \lambda_{5}\right\}\right. \\
&\left.-\left\{\lambda_{2}, \lambda_{3}, \lambda_{1}, \lambda_{4}, \lambda_{5}\right\}\right]=0,
\end{aligned}
$$

for $g$ the octic group generated by $x z, y u$; for $g$ the tetrahedral group generated by $x y z, x y \cdot z u$, a precisely similar $\iint$ with the suffixes of the respective \{\}, in this order, 23145, 13425, 23145; for $g$ the octahedral group generated by $x y, y z u$, the like with suffixes $21345,32415,23145$; and for $g$ the icosahedral 
group generated by $x y \cdot z u, x z v$, the like with suffixes 21435, 21543, 23145.

(ix) As a numerical example, we take the invariant, from the same source as (vi), for $g=1, x y$, and $\{x, y\} \equiv(\mid(x, y))^{g}$, $\iint\left[2(-1)^{\lambda}\left\{x_{1}+z_{1}, y_{1}\right\}+(-1)^{\mu}\left\{\frac{x_{2}+y_{2}}{2}+z_{2}, z_{2}\right\}\right]=0$; $\Sigma: 2 m=2 x_{1} y_{1}+4 z_{1}^{2}=x_{2} y_{2}+z_{2}^{2}$,

$m$ odd, $x_{1}, y_{1}, x_{2}, y_{2}$ odd $>0, z_{1}$ arbitrary $0 ; z_{2}$ odd $\gtreqless 0$; $\lambda \equiv z_{1}, 2 \mu \equiv y_{2}+z_{2}$. Let $m=5$; then all the solutions of $\Sigma$ are

$\left(x_{1}, y_{1}, z_{1}\right)=(1,5,0),(5,1,0),(1,3, \pm 1),(3,1, \pm 1)$;

$\left(x_{2}, y_{2}, z_{2}\right)=(1,9, \pm 1),(9,1, \pm 1),(1,1, \pm 3),(3,3, \pm 1)$.

Whence, substituting in the left of the invariant, and dropping the $\int \mathcal{S}$, we get

$$
\begin{aligned}
& 2[\{1,5\}+\{5,1\}-\{3,3\}-\{5,1\}-\{-1,3\}-\{1,1\}] \\
& +[-\{-3,1\}+\{1,1\}-\{5,1\}+\{-5,-1\} \\
& -\{-1,-1\}+\{3,-1\}+\{3,3\}-\{-3,-3\}] .
\end{aligned}
$$

Since $\{x, y\}$ belongs to $g,\{x, y\}=\{y, x\}$; hence the sum reduces to

$$
\begin{aligned}
{[\{1,5\}+\{-1,-5\}]-[\{3,3\}+\{-3,-3\}] } \\
-[\{-1,3\}+\{1,-3\}]-[\{1,1\}+\{-1,-1\}] .
\end{aligned}
$$

By $\S 12$ (II) this should vanish when $\{x, y\}$ is any one* of $(\mid(x, y)),(x \mid y),(y \mid x)$ each belonging to $g$. Taking the first of these we have $\{-1,-5\} \equiv(\mid(-1,-5))=-(\mid(1,5))$, so that the first [ ] $=0$; similarly in this case for the others. Verifying for $(x \mid y)$, we have $(-1 \mid-5)=-(1 \mid 5)$; hence the first []$=0$; the third $=(-1 \mid 3)+(1 \mid-3)=(1 \mid 3)$ $-(1 \mid 3)=0 ;$ the fourth $=(1 \mid 1)+(-1 \mid-1)=(1 \mid 1)$

$-(1 \mid 1)=0$. The verification for $(y \mid x)$ is similar. UNIVERSITY OF WASHINGTON.

* The cases of $(x \mid y),(y \mid x)$ are trivial in this instance, because it is readily seen that each vanishes under the given conditions for all values of $x, y$. 